\title{
Postoperative infections: Aetiology, incidence and risk factors among neurosurgical patients in Mthatha, South Africa
}

C Bokop Fotso, ${ }^{1}$ BSc Hons, MSc; D T Abaver, ${ }^{1}$ BSc, MSc, PhD; D Muballe, ${ }^{2}$ PhD; $\mathbf{S}$ Vasaikar, ${ }^{1,3}$ MB BS, MD (Medical Microbiology), PhD; T Apalata, ${ }^{1,3}$ MMed (Medical Microbiology), MBA, FICR (Global Health), PhD

\author{
${ }^{1}$ Division of Medical Microbiology, Department of Laboratory Medicine and Pathology, Faculty of Health Sciences, Walter Sisulu University, \\ Mthatha, South Africa \\ ${ }^{2}$ Department of Neurosurgery, Nelson Mandela Academic Complex and Faculty of Health Sciences, Walter Sisulu University, Mthatha, South Africa \\ ${ }^{3}$ National Health Laboratory Service, Mthatha, South Africa
}

Corresponding author: D T Abaver (dominicabaver@yahoo.com)

\begin{abstract}
Background. Despite progress in hospital care, infections continue to represent one of the major complications among hospitalised patients. Objectives. To determine the aetiology and incidence of hospital-acquired infections and their associated risk factors following neurosurgical procedures.

Methods. A retrospective study was conducted from October 2013 to September 2014. Data including demographics, hospitalisation period, type of operation and primary diagnosis were collected. Post-surgical infections were confirmed microbiologically. SPSS (Statistical Package for the Social Sciences) version 23 was used for statistical analysis.

Results. Among a total of 1688 patients who underwent neurosurgical operations, the incidence of post-surgical infections was $4.2 \%$ per year. Post-surgical infections were significantly associated with craniotomy $(p<0.0001)$, prolonged stay in hospital $(\geq 30$ days $)(p=0.008)$, and patient age $\geq 35$ years $(p=0.05)$. Staphylococcus aureus was the most frequently isolated pathogen $(19.7 \%)$, followed by Klebsiella pneumoniae (12.7\%). A total of $42.9 \%$ of S. aureus isolates were methicillin-resistant S. aureus (MRSA), but all these isolates were susceptible to vancomycin; $44.4 \%$ of $K$. pneumoniae isolates were extended-spectrum beta-lactamase (ESBL)-positive, but were susceptible to carbapenems, piperacillin-tazobactam and amikacin.

Conclusions. Post-surgical infections remain an important problem in neurosurgery. Increased resistance to causative pathogens is a major concern.
\end{abstract}

S Afr Med J 2020;110(5):403-408. https://doi.org/10.7196/SAMJ.2020.v110i5.13779

Healthcare-associated infections, also known as nosocomial infections, which affect patients in a hospital or healthcare facility and were not present or incubating at the time of admission, ${ }^{[1]}$ are among the main patient safety challenges in healthcare facilities. ${ }^{[2]}$ They can be defined as an infection that develops 48 hours after hospital admission or within 48 hours after discharge. ${ }^{[3]}$ In spite of improvements in healthcare, nosocomial infections continue and can be acquired anywhere healthcare is delivered, including inpatient acute-care hospitals, outpatient settings such as ambulatory surgical centres, end-stage renal disease facilities, and long-term care facilities such as nursing homes and rehabilitation centres. The development of nosocomial infections is dependent on two key pathophysiological factors: decreased host defences and colonisation by pathogenic or potentially pathogenic organisms. ${ }^{[4]}$ A survey published by the New England Journal of Medicine (cited in Becker's Clinical Leadership and Infection Control ${ }^{[5]}$ ) provides important insight for healthcare providers in their efforts to combat infections, reporting prevalence of the five commonest recorded nosocomial infections as follows: pneumonia $21.8 \%$ of all healthcare-associated infections; surgical site infection (SSI) 21.8\%; gastrointestinal infection $17.1 \%$; urinary tract infection $12.9 \%$; and primary bloodstream infection $9.9 \%$. It is estimated that $\sim 1$ in 7 patients entering South African (SA) hospitals is at high risk of acquiring a nosocomial infection. ${ }^{[6]}$ Neurosurgical patients are particularly vulnerable because of the serious nature of their illnesses, the frequency of associated trauma, and the presence of invasive devices. Neurosurgeons treat conditions such as aneurysms, stroke, epilepsy, meningitis, traumatic brain injury, spinal cord injury and brain tumours, all of which predispose patients to fungal, viral and bacterial infections. A study conducted in Pretoria, SA, reported that the incidence of ventriculitis reached $28.3 \%$ among paediatric neurosurgical patients. ${ }^{[7]}$

\section{Objectives}

Nelson Mandela Academic Hospital (NMAH) in Mthatha, like many hospitals in $\mathrm{SA},{ }^{[7]}$ faces high rates of nosocomial infections. The present study sought to determine the aetiology and incidence of these infections following neurosurgical procedures, as well as the associated risk factors.

\section{Methods}

This was a descriptive cross-sectional study. Patients were enrolled from 1 October 2013 to 30 September 2014. All inpatients who had had a neurosurgical procedure at NMAH during the study period were included in the study if their medical and laboratory records were accurate, legible and complete. Medical records were accessed in the Department of Neurosurgery at NMAH where patients were admitted, while laboratory records were obtained from the Department of Medical Microbiology at the National Health 
Laboratory Service (NHLS), NMAH. Accuracy of records was assessed in terms of precision of information or instructions related to the patient and his/her condition. Legibility of records was assessed in terms of clarity and readability, while completeness of records was assessed in terms of completeness of information or instructions provided in the records.

Clinical samples (blood, pus, pus swabs, cerebrospinal fluid (CSF), catheter tips, wound aspirates and tissues) were collected by medical doctors in the neurosurgical unit as part of routine patient management and submitted to the Department of Medical Microbiology at the NHLS for analysis.

The isolates were then stored in skim-milk agar and kept at $-20^{\circ} \mathrm{C}$ until further use (for identification and antimicrobial susceptibility testing). They were then subcultured into nutrient agar and McConkey agar and incubated for 24 hours at $37^{\circ} \mathrm{C}$.

The API-20E test kit (bioMérieux, USA) was used for identification of members of the family Enterobacteriaceae and associated nonfastidious organisms. The oxidase test was used to identify bacteria that produce cytochrome $\mathrm{C}$ oxidase, an enzyme of the bacterial electron transport chain. The test is used in differentiating between the Enterobacteriaceae and pseudomonads, which are oxidasenegative and oxidase-positive, respectively. An analytical profile index for species identification (RAPIDEC STAPH, bioMérieux, USA) was used to identify staphylococci and micrococci, and a salt tolerance test was used to identify enterococcal group D streptococci on the basis of their salt tolerance.

MicroScan (WalkAway 96 IS system; Siemens, UK) panels were used for further identification of micro-organisms, as follows: modified conventional and chromogenic tests were used to identify fermentative and non-fermentative Gram-negative bacilli. Identification was based on detection of $\mathrm{pH}$ changes, substrate utilisation, and growth in the presence of antimicrobial agents after 16 - 42 hours' incubation at $35^{\circ} \mathrm{C}$.

In this study, we measured the incidence proportion, defined as the measure of the risk of developing a new neurosurgical infection within a 12-month period. To calculate the incidence proportion, the numerator was the number of disease onsets (all new cases of postsurgical infection) and the denominator was the number of patients initially at risk (all patients who underwent a neurosurgical procedure).

To determine risk factors associated with neurosurgical infections, clinical information was collected from patients, including demographic characteristics and clinical status (such as underlying diseases). Data on microbial treatment of micro-organisms were recorded, including antibiotic, dose administered, routes of administration, duration of therapy, and dates of admission, surgery and discharge.

Wound class, neurosurgical procedure, antibiotic use, infecting pathogens, severity of illness and preoperative time were recorded. In the event of infection, detailed records were collected regarding treatment provided and patient outcome, as well as the type of invasive device used during neurosurgery.

\section{Statistical analysis}

Continuous variables were expressed as means and standard error of the mean (SEM), and categorical variables were expressed as proportions. The level of significance was set at $p<0.05$. Chi-square analysis was performed to determine risk factors associated with neurosurgical infections. The Statistical Package for the Social Sciences (SPSS), version 23 (IBM, USA), was used for analysis.

\section{Ethical considerations}

Ethical clearance was obtained from the Walter Sisulu University Ethics and Biosafety Committee (ref. no. 071/2014). Since this study included a descriptive survey of clinical and laboratory records, permission to access those records was obtained from the hospital management at NMAH and also from the NHLS laboratory manager.

\section{Results}

Between 1 October 2013 and 30 September 2014, 1688 patients were admitted for neurosurgical procedures at NMAH. Of these patients, $1266(75.0 \%)$ were male and $422(25.0 \%)$ were female. The mean (SEM) age of the male patients was 17.2 (2.6) years and that of the female patients 17.5 (6.4) years.

Of the patients, $127(7.5 \%)$ were clinically suspected to have developed a nosocomial infection, but only 71 cases were confirmed microbiologically $(71 / 1688,4.2 \%)$. Of the 127 patients with suspected infection, $88(69.3 \%)$ were male and $39(30.7 \%)$ were female. The mean (SEM) age of adult patients was 22.8 (2.0) years, while infants and neonates had mean ages of 8.14 (1.4) months and 37 (16) days, respectively (Table 1).

Of the 127 clinically suspected post-surgical infections, 61 occurred after craniotomy and 66 after a ventricular shunt. The commonest underlying neurosurgical abnormalities were hydrocephalus (48.8\%), traumatic brain injury (33.1\%) and brain abscess (9.4\%), as shown in Table 1

Of 127 clinically suspected cases of nosocomial infection, 61 (48.0\%) and $10(7.9 \%)$ were microbiologically confirmed as SSIs and central nervous system infections (CNSIs), respectively. CNSIs included meningitis and ventriculoperitoneal (VP) shunt infection.

The results showed that craniotomy was significantly associated with SSI rather than CNSI; on the other hand, the presence of VP shunt was significantly associated with CNSI rather than SSI ( $p=0.001$ ). When we compared patients with and without confirmed nosocomial infections ( $n=71$ and $n=56$, respectively), traumatic

Table 1. Baseline characteristics of patients diagnosed with nosocomial infections based on clinical suspicion during the study period $(N=127)$

\begin{tabular}{ll}
\hline Age, mean (SEM) & $22.8(2.0)$ \\
Adults (years) $(n=97)$ & $8.1(1.4)$ \\
Infants (months) $(n=28)$ & $37(16)$ \\
Neonates (days) $(n=2)$ & \\
Gender, $n$ (\%) & $88(69.3)$ \\
$\quad$ Male & $39(30.7)$ \\
Female & \\
Neurosurgical abnormality, $n(\%)$ & $3(2.4)$ \\
Intracranial haemorrhage & $42(33.1)$ \\
Traumatic brain injury & $62(48.8)$ \\
Hydrocephalus & $12(9.4)$ \\
Brain abscess & $4(3.1)$ \\
Brain tumour & $3(2.4)$ \\
Subdural empyema & $1(0.8)$ \\
Lumbar spine injury & \\
Surgical characteristics, $n(\%)$ & $61(48.0)$ \\
Craniotomy & $65(51.2)$ \\
Ventriculoperitoneal shunt & $1(0.8)$ \\
Spinal cord operation & \\
Nosocomial infections, $n$ (\%) & $61(48.0)$ \\
Surgical site infection & $1(0.8)$ \\
Meningitis & $9(7.1)$ \\
Cerebrospinal shunt infection & $56(44.1)$ \\
No microbial confirmation & \\
SEM = standard error of the mean. & \\
\end{tabular}


brain injury and brain abscess were significantly associated with the presence of post-surgical infection, while hydrocephalus was significantly associated with absence of post-surgical infection $(p<0.0001)$. Post-surgical infections were significantly associated with craniotomy $(p<0.0001)$, prolonged stay in hospital ( $>30$ days) ( $p=0.008)$ and patient age $\geq 35$ years $(p=0.05)$ (Table 2$)$.

Length of hospital stay $>30$ days was significantly associated with post-surgical infection $(p=0.025)$, as shown in Fig. 1. To determine length of stay as a risk factor in our study, we divided the numbers of days spent in the ward into three categories: $<14$ days, $14-30$ days and $>30$ days. As shown in Fig. 1, the longer a patient spent in hospital, the higher the risk became of developing SSI or CNSI.

The associations between post-surgical nosocomial infection and the duration of urinary, endotracheal and intravenous catheter placement did not reach statistical significance (data not shown).

The commonest aetiological agents isolated from the 71 culturepositive patients were Staphylococcus aureus $(n=14 ; 19.7 \%)$ and Klebsiella pneumoniae $(n=9 ; 12.7 \%)$ (Table 3$)$. The most frequently isolated Gram-positive bacteria were S. aureus (19.7\%), Enterococcus faecalis (11.3\%) and S. epidermidis (9.9\%), while the most frequently isolated Gram-negative bacteria were K. pneumoniae (12.7\%), Acinetobacter baumannii (8.5\%), Escherichia coli (8.5\%) and Proteus mirabilis (8.5\%) (Table 3).

Of 14 isolates of $S$. aureus, 6 (42.9\%) were methicillin-resistant S. aureus (MRSA); however, all were susceptible to vancomycin. The remaining 8 non-MRSA isolates $(57.1 \%)$ were also susceptible to erythromycin and clindamycin, but all $S$. aureus isolates were resistant to benzylpenicillin and ampicillin. All 7 isolates of $S$. epidermidis were resistant to benzylpenicillin, ampicillin, erythromycin and clindamycin, while $4(57.1 \%)$ were resistant to methicillin. Of the 8 isolates of E. faecalis, 3 (37.5\%) were vancomycin resistant and also displayed high-level gentamicin resistance. The remaining isolates were all susceptible to ampicillin and benzylpenicillin. All isolates of streptococci (either S. milleri or Streptococcus spp.) were susceptible to benzylpenicillin, ampicillin, erythromycin, clindamycin and vancomycin.

All the Gram-negative isolates considered resistant were extendedspectrum beta-lactamase (ESBL) producers, and all were resistant to third-generation cephalosporins (ceftriaxone, cefotaxime and ceftazidime) and gentamicin, but all these ESBL isolates were susceptible to carbapenems (imipenem, meropenem and ertapenem), piperacillin-tazobactam and amikacin. All ESBL-producing K. pneumoniae and Enterobacter cloacae were also resistant to ciprofloxacin. As shown in Table 3, 4/9 K. pneumoniae (44.4\%), 4/6 A. baumannii (66.7\%), 2/6 E. coli (33.3\%) and P. mirabilis (33.3\%), and $2 / 3$ E. cloacae $(66.7 \%)$ were ESBL producers. All isolates

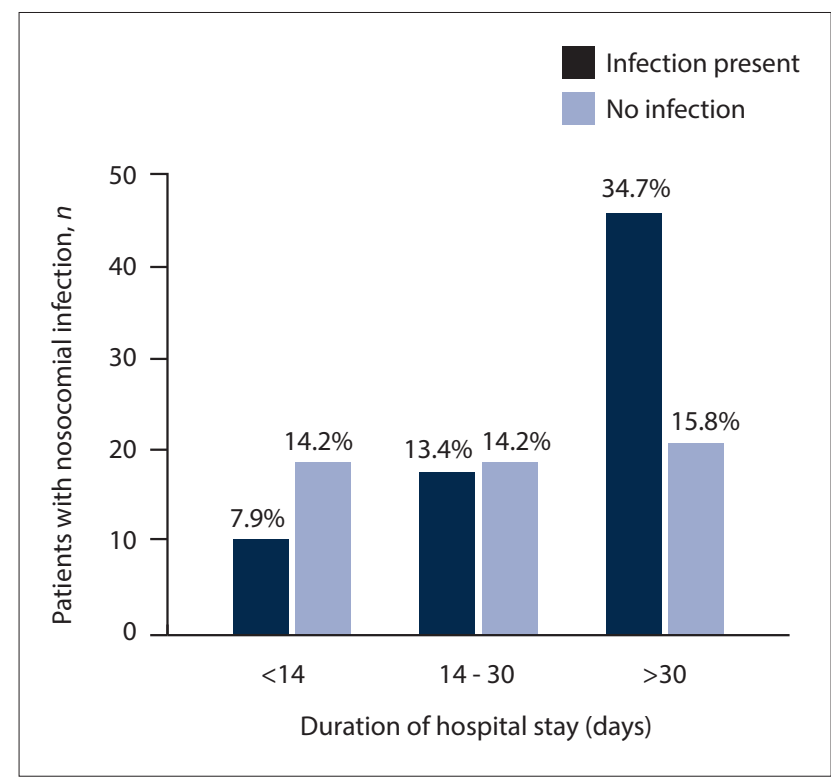

Fig. 1. Rates of nosocomial infection in relation to length of stay.

\begin{tabular}{|c|c|c|c|}
\hline Variables of interest & Presence of infection $(N=71), n(\%)$ & Absence of infection $(N=56), n(\%)$ & $p$-value \\
\hline Gender & & & 0.30 \\
\hline Male & $51(58.0)$ & $37(42.0)$ & \\
\hline Female & $20(51.3)$ & $19(48.7)$ & \\
\hline Age (years) & & & 0.05 \\
\hline$<35$ & $53(52.0)$ & $49(48.0)$ & \\
\hline$\geq 35$ & $18(72.0)$ & $7(28.0)$ & \\
\hline Surgical procedure & & & $<0.0001$ \\
\hline Craniotomy & $57(93.4)$ & $4(6.6)$ & \\
\hline VP shunt & $14(21.2)$ & $52(78.8)$ & \\
\hline Length of stay (days) & & & 0.008 \\
\hline$<14$ & $10(35.7)$ & $18(64.3)$ & \\
\hline $14-30$ & $17(48.6)$ & $18(51.4)$ & \\
\hline$>30$ & $44(68.8)$ & $20(31.2)$ & \\
\hline Primary diagnosis & & & $<0.0001$ \\
\hline Traumatic brain injury & $41(97.6)$ & $1(2.4)$ & \\
\hline Hydrocephalus & $13(21.0)$ & $49(79.0)$ & \\
\hline Brain abscess & $9(75.0)$ & $3(25.0)$ & \\
\hline Brain tumour & $2(50.0)$ & $2(50.0)$ & \\
\hline Lumbar spine injury & $1(100)$ & 0 & \\
\hline Intracranial haemorrhage & $3(100)$ & 0 & \\
\hline Subdural empyema & $2(66.7)$ & $1(33.3)$ & \\
\hline
\end{tabular}


Table 3. Frequency of isolation and antibiotic resistance of micro-organisms isolated from 71 culture-positive patients

\begin{tabular}{|c|c|c|}
\hline Micro-organisms isolated & Isolates, $\boldsymbol{n}$ (\% of total isolates) & $\begin{array}{l}\text { Resistant isolates, } n \text { (\% of total } \\
\text { isolates of a particular organism) }\end{array}$ \\
\hline \multicolumn{3}{|l|}{ Gram-positive bacteria } \\
\hline Staphylococcus aureus* & $14(19.7)$ & $6(42.9)$ \\
\hline Enterococcus faecalis ${ }^{\dagger}$ & $8(11.3)$ & $3(37.5)$ \\
\hline S. epidermidis ${ }^{\ddagger}$ & $7(9.9)$ & $4(57.1)$ \\
\hline Streptococcus milleri ${ }^{\Im}$ & $2(2.8)$ & 0 \\
\hline Streptococcus spp. ${ }^{\varsigma}$ & $2(2.8)$ & 0 \\
\hline \multicolumn{3}{|l|}{ Gram-negative bacteria } \\
\hline Klebsiella pneumoniaes & $9(12.7)$ & $4(44.4)$ \\
\hline Acinetobacter baumannii & $6(8.5)$ & $4(66.7)$ \\
\hline Escherichia coli & $6(8.5)$ & $2(33.3)$ \\
\hline Proteus mirabilis & $6(8.5)$ & $2(33.3)$ \\
\hline Enterobacter cloacaes & $3(4.2)$ & $2(66.7)$ \\
\hline Pseudomonas aeruginosa" & $2(2.8)$ & 0 \\
\hline Others ${ }^{* *}$ & $6(8.5)$ & 0 \\
\hline \multicolumn{3}{|c|}{ 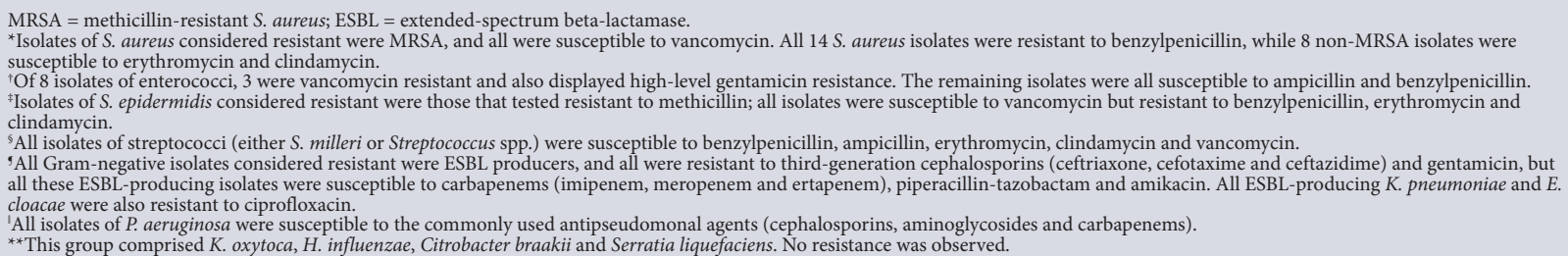 } \\
\hline
\end{tabular}

of Pseudomonas aeruginosa were susceptible to the commonly used antipseudomonal agents (cephalosporins, aminoglycosides and carbapenems) (Table 3).

\section{Discussion}

Despite all efforts proposed by infection prevention and control guidelines to control nosocomial infections through improvements in operating rooms, ventilation, sterilisation methods, barriers and better surgical techniques, complete elimination of nosocomial infections in neurosurgical patients remains a challenging goal, and these infections are still one of the most important causes of mortality in hospitalised patients. ${ }^{[8]}$

\section{Rates of nosocomial infections in neurosurgical patients} The overall incidence of nosocomial infection after neurosurgical operations in the present study was $7.5 \%$ based on clinical suspicion and $4.2 \%$ based on microbiological confirmation. Bellusse et al. ${ }^{[9]}$ conducted a study on infections following neurosurgical procedures in a Brazilian tertiary hospital and found a $7.7 \%$ incidence rate of nosocomial infection. A study by Buang and Haspani ${ }^{[10]}$ had similar results, with a $7.7 \%$ rate of nosocomial infections in Kuala Lumpur Hospital, Malaysia.

Analysis of types of postoperative infections seen in the present study revealed higher rates of SSIs compared with CNSIs (VP shunt infection and meningitis). Similar findings were observed by McClelland and Hall ${ }^{[1]}$ in a study of patients in a tertiary hospital at the University of Minnesota (Minneapolis).

Of the patients who developed nosocomial infection in the present study, $69.3 \%$ were male and $30.7 \%$ were female. These figures correspond with those reported by Buang and Haspani ${ }^{[10]}$ (69.5\% male and $30.5 \%$ female). Other studies also show that males have a higher predisposition to nosocomial infections than females. ${ }^{[12]}$ This increased risk may be because males of middle age are more likely than females to engage in risk behaviours such as drinking excessively, driving while drunk, getting involved in physical fights and other risky practices that render them susceptible to accidents. Our findings show that men with such a lifestyle tend to be unmarried, to live alone, not to be well educated, and to be unemployed.

\section{Factors associated with nosocomial infections in neurosurgical patients}

The present study found that a variety of risk factors were associated with neurosurgical infections. The main risk factors were surgical procedure (craniotomy), primary diagnosis (traumatic brain injury and brain abscess), length of hospital stay ( $>30$ days) and patient age ( $\geq 35$ years).

We found that craniotomy was a risk factor associated with SSI rather than VP shunt, and that patients who underwent craniotomy were more likely to develop SSI than those with VP shunt operations (indicated for hydrocephalus). Kassam et al. ${ }^{[13]}$ in their study in Israel reported that the presence of a CSF leak and subsequent operation were risk factors for CNSI (mainly meningitis), while emergency surgery, clean-contaminated and dirty surgery, operative time $>4$ hours, and recent neurosurgery were found to be independent predictive risk factors for SSI. ${ }^{[9]}$ Blomsted ${ }^{[14]}$ found that postoperative CSF leak was the only highly significant risk factor for SSI. Other studies have shown that longer duration of surgery increased the incidence of SSI. ${ }^{[15]}$

Govender et al. ${ }^{[16]}$ stated that although development of effective CSF shunts represented a landmark achievement in neurosurgery, once the shunt is inserted, the patient is susceptible to a whole new disease entity together with its potential complications. It has also been postulated that the CSF shunt apparatus itself diminishes the host's defences at the site of implantation and increases the likelihood that an inoculum of bacteria will progress to an infection. ${ }^{[7]}$ In the present study, $83.3 \%$ 
of patients with VP shunt infection were aged $<2$ years. Similar results were found in a study by Hasanain et al. ${ }^{[17]}$ which showed that VP shunt failure was very common in infants, and that the most common cause of VP shunt failure in children $<2$ years was shunt infection.

Many studies have described specific evidence reported from the literature suggesting that the risk of infection increases when extraventricular devices (EVDs) such as VP shunts are retained for $>5$ days ${ }^{[18]}$ and $>10$ days. ${ }^{[19]}$ The present study showed that length of hospital stay was a risk factor for both SSI and CNSI, with mean (standard deviation) length of stay being 39.29 (3.60) days (median 33 days). Korinek et al. ${ }^{[20]}$ found that the mean time between surgery and the onset of SSI was 18 (25) days, while Buang and Haspani ${ }^{[10]}$ found it to be 11.8 (22.8) days (median 10 days) ${ }^{[10]}$ Liu et al ${ }^{[21]}$ suggested that shorter antimicrobial prophylaxis may increase post-neurosurgical infection. Another study reported that long duration of use of the drainage tube was likely to be an important factor causing postoperative central nervous system infection. ${ }^{[22]}$ Other factors considered in the present study were gender, age, duration of urinary and endotracheal catheterisation, ventilator-associated pneumonia, and presence of an intraventricular drain. Except for age ( $\geq 35$ years), none of these factors showed significant associations with either SSI or CNSI and they were therefore not considered as risk factors.

\section{Antimicrobial susceptibility profiles among the culture-positive isolates}

Among the Gram-positive bacteria, the most predominant virulent organism in our study was $S$. aureus, followed by $S$. epidermidis. Buang and Haspani ${ }^{[10]}$ also reported virulence of $S$. aureus, as well as MRSA, which were the most common pathogens causing neurosurgical infections. Other studies such as those by Negi et al. ${ }^{[23]}$ and Whitby et al. ${ }^{[2]}$ found that Gram-positive cocci were the main causative organisms responsible for surgical site infections among neurosurgical patients.

Forty-three percent of $S$. aureus isolated in this study were resistant to cloxacillin (MRSA), the first-line drug used in the management of staphylococcal infections in our setting. However, all tested MRSA isolates from our study were susceptible to vancomycin. Methicillin resistance in $S$. aureus is mediated by the mecA gene, which encodes for a novel penicillin-binding protein $2 \mathrm{a}$ (PBP-2a). ${ }^{[25]}$ In MRSA, exposure to methicillin inactivates the four high binding affinity PBPs normally present. PBP-2a, which displays a low affinity for methicillin, takes over the functions of these PBPs, permitting the cell to grow. ${ }^{[26]}$ Vancomycin remains the drug of choice for treatment of infection caused by MRSA, although it is thought to be intrinsically less active than the antistaphylococcal penicillin. ${ }^{[26]}$

S. epidermidis, commonly considered to be a harmless inhabitant of the human skin and mucous surfaces, is becoming one of the main causes of healthcare-acquired infections. In general terms, infections by $S$. epidermidis are usually characterised by the formation of biofilms (an aggregate of microbes that does not require many nutrients to survive with a distinct architecture), which frequently develop and grow on implants used in the hospital setting. The ability to form biofilms on plastic devices by producing an extracellular material known as polysaccharide intercellular adhesion, which is made up of sulphated polysaccharides, is a major virulence factor for S. epidermidis. The formed biofilm decreases the metabolic activity of bacteria. The presence of sulphated polysaccharides in combination with impaired diffusion of antibiotics makes it difficult for antibiotics to clear the infection effectively unless the device is removed.

Of the total isolates of K. pneumoniae in our study, $44.4 \%$ were ESBL producers but were susceptible to carbapenems, piperacillin- tazobactam and amikacin. ESBLs are $\beta$-lactamases capable of making bacteria resistant to penicillin, the first-, second- and thirdgeneration cephalosporins, and aztreonam (but not the cephamycins or carpenems) by hydrolysing these antibiotics. ${ }^{[27]}$ Such ESBLs may develop when antibiotics are taken for longer than necessary or when they are not needed. ${ }^{[27]}$ Apart from K. pneumoniae, ESBLs in our study were also identified in A. baumannii and E. cloacae. All isolates of $P$. aeruginosa, however, were susceptible to the commonly used antipseudomonal agents. Others have reported similar susceptibilities to those in our study. ${ }^{[28]}$ Moodley et al. ${ }^{[29]}$ stated that the majority of A. baumannii isolated in their study were multidrug-resistant, retaining susceptibility only to tobramycin and colistin. A study by Karlowsky et al. ${ }^{\left[{ }^{[0]}\right]}$ demonstrated that $>90 \%$ of A. baumannii isolates tested were susceptible to imipenem and meropenem. ${ }^{[28]}$

The increased resistance of $A$. baumannii to numerous antimicrobial agents is notable and has prompted its classification as a highpriority pathogen by the Antimicrobial Availability Task Force of the Infectious Diseases Society of America (as reported by Moodley et al. $\left.{ }^{[29]}\right)$. Jocum ${ }^{[31]}$ and Harbath et al. ${ }^{[32]}$ both reported that prolonged antibiotic prophylaxis was correlated with an increased risk of acquired antibiotic resistance. Possible other reasons for increased resistance of A. baumannii include excessive use of antimicrobials for acutely ill and complex patients in hospitals, diagnostic uncertainty, pressure to keep length of stay short, and prescription of antibiotics by inexperienced doctors, e.g. in teaching hospitals. ${ }^{[29]}$

In a recent international survey of neurosurgeons and critical care and infectious disease specialists, 56.3\% of neurosurgeons used prophylaxis as long as the EVD was in place, while only $11.5 \%$ of infectious disease specialists were in favour of doing so. ${ }^{[33]}$

The potential for survival of a microbe lies in its ability to change. Microbes are able to reproduce much faster than human cells. Bacterial cells replicate every 15 minutes, and each time a bacterium divides, there is a chance for error. A random change in the genetic makeup of a cell becomes a permanent inherited characteristic. ${ }^{[26]}$ Use of antibiotics creates a situation in which the fittest microbes survive.

\section{Study limitations}

One of the key limitations of this study was identification of risk factors by using patients clinically suspected to have postoperative infections who were found to be culture-negative (microbiologically not confirmed) as a control group (as opposed to patients who were microbiologically confirmed). This is because patients who were clinically suspected were not tested for the presence of anaerobic bacteria, fungi and other fastidious micro-organisms, as this was beyond the scope of this study.

\section{Recommendations}

Findings from this study support the following recommendations:

- Appropriate use of antimicrobial agents for prophylaxis and treatment. Since half of the isolated S. aureus and S. epidermidis, the prevalent Gram-positive bacteria, were resistant to first-line cloxacillin, and half of the commonly isolated Gram-negative bacteria were ESBL producers, we recommend that doctors should consider covering for MRSA and ESBL-producing organisms in any empirical treatment prescribed.

- Owing to increasing rates of multidrug-resistant organisms in neurosurgery, appropriate clinical samples must be collected prior to empirical antibiotic initiation and sent to the microbiology laboratory without any delay. Once laboratory results are obtained, empirical treatment must be corrected. 
- There is an urgent need to strengthen infection prevention and control activities in the ward and in theatre.

- Finally, surveillance of nosocomial infections and antimicrobial susceptibility testing must be performed regularly to inform treatment and quality improvement mechanisms.

\section{Conclusions}

Nosocomial infections remain important adverse events in the Department of Neurosurgery at NMAH. The overall incidence of microbiologically confirmed nosocomial infections was $4.2 \%$, despite the high rate of culture-negative results. This figure may be an underestimation of the current situation considering that the present study did not take into account cultures of anaerobic bacteria, fungi and other fastidious micro-organisms.

To minimise post-surgical infections, a combination of preventive practices should be used. These measures include a preoperative bath, appropriate antiseptic agents (chlorhexidine or povidone-iodine) for skin preparation preoperatively, and use of appropriate wound dressings postoperatively.

The increasing level of antimicrobial resistance in both Grampositive (MRSA and emerging coagulase-negative staphylococci) and Gram-negative (ESBL-positive K. pneumoniae and emerging multidrug-resistant Acinetobacter species) bacteria in neurosurgery is grounds for grave concern and requires urgent attention. Appropriate use of antimicrobial agents for both prophylactic and therapeutic administration remains the first step towards overcoming the challenge of multidrug-resistant organisms.

Declaration. The research for this study was done in partial fulfilment of the requirements for CBF's BSc Hons degree in the Department of Medical Microbiology, Walter Sisulu University.

Acknowledgements. None.

Author contributions. CBF: study concept and data collection; DTA: data analysis, manuscript development and editing of the final manuscript; DM: data collection; SV: data analysis and editing of the final manuscript; TA: concept, protocol development, manuscript development, data analysis and editing of the final manuscript.

Funding. None.

Conflicts of interest. None.

1. Montana State Hospital Policy and Procedure: Health Care Associated Infections. Policy: IC-13. 12 January 2016. https://dphhs.mt.gov/Portals/85/amdd/documents/MSH/volumei/infectioncontrol/ Health CareAssociatedInfections. pdf (accessed 15 February 2019).

2. Ferreira E, Pina E, Sousa-Uva M, Sousa-Uva A. Risk factors for health care-associated infections From better knowledge to better prevention. Am J Infect Control 2017;45(10):e103-e107. https://doi. org $/ 10.1016 /$ jaic 2017.03 .036

3. Stubblefield $\mathrm{H}$, medically reviewed by Rogers $\mathrm{G}$. What are nosocomial infections? Healthline, 24 October 2016. https://www.healthline.com/health/hospital-acquired-nosocomial-infections (accessed 16 February 2019).

4. Custovic A, Smajlovic J, Hadzic S, Ahmetagic S, Tihic N, Hadzagic H. Epidemiological surveillance of bacterial nosocomial infections in the surgical intensive care unit. Mater Sociomed 2014;26(1):7-11 https://doi.org/10.5455/msm.2014.26.7-11

5. Becker's Clinical Leadership and Infection Control. 13 most common healthcare-associated infections. 4 September 2014. https://www.beckershospitalreview.com/quality/13-most-common-healthcareassociated-infections.html (accessed 16 February 2019).
6. Patel B, Engelbrecht H, McDonald H, Morris V, Smythe W. A multifaceted hospital-wide intervention increases hand hygiene compliance. S Afr Med J 2016;106(4):32-35. https://doi.org/10.7196/ AMJ.2016.v106i4.10671

7. Motloba DP, Ngqandu MD. Ventriculostomy infections at the paediatric neurosurgical unit at Dr George Mukhari Academic Hospital. S Afr J Child Health 2015;9(1):6-8. https://doi.org/10.7196/ SAJCH.767

8. Hassan AK, Fatima KB, Riffat M. Nosocomial infections: Epidemiology, prevention, control and surveillance. Asian Pac J Trop Biomed 2017;7(5):478-482. https://doi.org/10.1016/j.apjtb.2017.01.019 Bellusse GC, Ribeiro JC, Fabrício RC, Poveda VD, Galvão CM. Risk factors for surgical site infection in neurosurgery. Ann R Coll Surg Engl 2015;28(1):66-73. https://doi.org/10.1308/rcsann.2019.0001

0. Buang SS, Haspani MS. Risk factors for neurosurgical site infections after a neurosurgical procedure: A prospective observational study at Hospital Kuala Lumpur. Med J Malaysia 2012;67(4):393-398.

11. McClelland S, Hall WA. Postoperative central nervous system infection: Incidence and associated factors in 2111 neurosurgical procedures. Clin Infect Dis 2007;45(1)1:55-59. https://doi. org $/ 10.1086 / 518580$

12. Taha MM, Abouhashem S, Abdel-rahman AY. Neurosurgery wound infection at a university hospital in Egypt: Prospective study of 1181 patients for 2 years. Turk Neurosurg 2014;24(1):8-12. https://doi. org/10.5137/1019-5149.JTN.6464-12.1

13. Kassam AB, Prevedello DM, Carrau RL, et al. Endoscopic endonasal skull base surgery: Analysis of complications in the authors' initial 800 patients. J Neurosurg 2011;114(6):1544-1568. https://doi. org/10.3171/2010.10.JNS09406

14. Blomstedt GC. Infections in neurosurgery: A retrospective study of 1143 patients and 1517 operations. Acta Neurochir (Wien) 1985;78(3-4):81-90. https:///doi.org/10.1007/bfo1808684

15. Fang C, Zhu T, Zhang P, Xia L, Sun C. Risk factors of neurosurgical site infection after craniotomy: A systematic review and meta-analysis. Am J Infect Control 2017;45(11):e123-e134. https://doi. org/10.1016/j.ajic.2017.06.009

16. Govender ST, Nathoo N, van Dellen JR. Evaluation of an antibiotic-impregnated shunt system for the treatment of hydrocephalus. J Neurosurg 2003;99(5):831-839. https://doi.org/10.3171/ ns.2003.99.5.083

17. Hasanain AA, Abdullah A, Alsawy MFM, et al. Incidence of and causes for ventriculoperitoneal shunt failure in children younger than 2 years: A systematic review. J Neurol Surg A Cent Eur Neurosurg 2019;80(01):26-33. https:doi.org/10.1055/s-0038-1669464

18. Kitchen WJ, Singh N, Hulme S, Galea J, Patel HC, King AT. External ventricular drain infection: Improved technique can reduce infection rates. Br J Neurosurg 2011;25(5):632-635. https://doi.org/ $10.3109 / 02688697.2011 .578770$

19. Holloway KL, Smith KW, Wilberger JE Jr, Jemsek JG, Giguere GC, Collins JJ. Antibiotic prophylaxis during clean neurosurgery: A large, multicenter study using cefuroxime. Clin Ther 1996;18(1):84-94. https://doi.org/10.1016/s0149-2918(96)80181-0

20. Korinek AM, Baugnon T, Golmard JL, van Effenterre R, Coriat P, Puybasset L. Risk factors for adult nosocomial meningitis after craniotomy: Role of antibiotic prophylaxis. Neurosurgery 2008;62(Suppl 2):126-133. https://doi.org/10.1227/01.neu.0000316256.44349.b1

21. Liu H, Wu YQ, Gao X, et al. Biofilm-related genes: Analyses in multi-antibiotic resistant Acinetobacter baumannii isolates from mainland China. Med Sci Monit 2016;22:1801-1807. https://doi.org/10.12659/ msm.898959

22. Hagel S, Bruns T, Pletz MW, et al. External ventricular drain infections: Risk factors and outcome. Interdiscip Perspect Infect Dis 2014;2014:708531. https://doi.org/10.1155/2014/708531

23. Negi V, Pal S, Juyal D, Sharma MK, Sharma N, Bacteriological profile of surgical site infections and their antibiogram: A study from resource constrained rural setting of Uttarakhand State, India. J Clin Diagn Res 2015;9(10):DC17-DC20. https://doi.org/10.7860/JCDR/2015/15342.6698

24. Whitby M, Johnson BC, Atkinson RL, Stuart G. The comparative efficacy of intravenous cefotaxime and trimethoprim/sulfamethoxazole in preventing infection after neurosurgery: A prospective, randomized study. Br J Neurosurg 2000;14(1):13-18. https://doi.org/10.1080/02688690042843

25. Alexander JAN, Chatterjee SS, Hamilton SM, Eltis LD, Chambers HF, Strynadka NCJ. Structural and kinetic analyses of penicillin-binding protein 4 (PBP4)-mediated antibiotic resistance in Staphylococcus aureus. J Biol Chem 2018;293(51):19854-19865. https://doi.org/10.1074/jbc.RA118.004952

26. Peacock SJ, Paterson GK. Mechanisms of methicillin resistance in Staphylococcus aureus. Annu Rev Biochem 2015;84:577-601. https://doi.org/10.1146/annurev-biochem-060614-034516

27. Rahman UR, Ali T, Ali I, Khan NA, Han B, Gao J. The growing genetic and functional diversity of extended spectrum beta-lactamases. Biomed Res Int 2018;2018:9519718. https://doi. org $/ 10.1155 / 2018 / 9519718$

28. Qureshi S. Pseudomonas aeruginosa infections medication. Medscape, 3 March 2018. https:// emedicine.medscape.com/article/226748-medication (accessed 10 February 2019).

29. Moodley VM, Oliver SP, Shankland I, Elisha BG. Evaluation of five susceptibility test methods for Moodley VM, Oliver SP, Shankland I, Elisha BG. Evaluation of five susceptibility test methods for
detection of tobramycin resistance in a cluster of epidemiologically related Acinetobacter baumannii detection of tobramycin resistance in a cluster of epidemiologically related Acinetobacter
isolates. J Clin Microbiol 2013;51(8):2535-2540. https://doi.org/10.1128/JCM.03250-12

30. Karlowsky JA, Draghi DC, Jones ME, Thornsberry C, Friedland IR, Sahm DF. Surveillance for antimicrobial susceptibility among clinical isolates of Pseudomonas aeruginosa and Acinetobacter baumannii from hospitalized patients in the United States, 1998 to 2001. Antimicrob Agents Chemother 2003;47(5):1681-1688. https://doi.org/10.1128/aac.47.5.1681-1688.2003

31. Jocum J. Surgical antibiotic prophylaxis: Are you doing it right? South Afr J Anaesth Analg 2018;24(3):S49-S5

32. Harbarth S, Samore MH, Lichtenberg D, Carmeli Y. Prolonged antibiotic prophylaxis after cardiovascular surgery and its effect on surgical site infections and antimicrobial resistance. Circulation 2000;101(25):2916-2921. https://doi.org/10.1161/01.cir.101.25.2916

33. McCarthy PJ, Patil S, Conrad SA, Scott LK. International and specialty trends in the use of prophylactic antibiotics to prevent infectious complications after insertion of external ventricular drainage devices. Neurocrit Care 2010;12(2):220-224. https://doi.org/10.1007/s12028-009-9284-

Accepted 13 September 2019 